\title{
Increasing the Resolution of Wide-Area Situational Awareness of the Power Grid through Event Unmixing
}

\author{
Hairong Qi*, Yilu Liu, Fran Li, Jiajia Luo, Li He, Kevin Tomsovic, Leon \\ Tolbert, Qing Cao \\ Electrical Engineering and Computer Science \\ University of Tennessee \\ Knoxville, TN 37996 \\ \{hqi, liu, fli6, jluo9, lhe4, tomsovic, tolbert, cao\}@utk. edu
}

\begin{abstract}
Energy infrastructure is a critical underpinning of modern society. To ensure its safe and healthy operation, a wide-area situational awareness system is essential to provide high-resolution understanding of the system dynamics such that proper actions can be taken in time in response to power system disturbances and to avoid cascading blackouts. This paper focusses on the high resolution or finer-scale analysis of data obtained through the North American frequency monitoring network (FNET) to reveal hidden information. In the power grid, events seldom occur in an isolated fashion. Cascading events and simultaneous events are more common and realistic. We present a new conceptual framework in event analysis, referred to as event unmixing, where we consider real-world events as a mixture of more than one constituent root event. This concept is a key enabler for the analysis of events to go beyond what are immediately detectable in the system. We interpret the event formation process from spectral mixing perspective and present innovative unsupervised unmixing algorithms to unravel complex events.
\end{abstract}

\section{INTRODUCTION}

With an estimated worth of over $\$ 800$ billion, the US electric power grid includes over 15,000 generators and hundreds of thousands of miles of transmission and distribution lines. Power grids are vital links that achieve the essential continuity of service from the generating plants to the end users. Wide-area situational awareness (WASA) systems enable the monitoring of overall bulk power systems and provide critical information for understanding and responding to power system disturbances and cascading blackouts.

The concept of "high-resolution" event analysis is twofold. From sensing perspective, high resolution is reflected in both high-precision measurement and potential for dense spatial coverage. From data analysis perspective, high resolution is to formulate the problem from a new perspective such that event formation can be studied at a much finer resolution to facilitate spatial-temporal situational awareness.

\section{A. Sensing}

To maintain power system operation in a balanced and stable condition, the frequency deviation and the rate of frequency change information are highly desired [16], [28] in monitoring and protection applications of the power grid. How to obtain frequency information more accurately and

\footnotetext{
*This work is supported in part by National Science Foundation under CNS-0831466.
}

efficiently has been the topic of discussion for decades. PMUs (Phasor Measurement Unit) [1], [5], [8], [32] are the most widely-used devices that calculate phase angle from voltage signal measurements. Since a Virginia Tech research team developed the first prototype PMU in 1988, PMUs have been gradually but cautiously deployed throughout the North American power system. At present, there are only 105 PMUs installed in the Eastern Interconnection (EI) and 56 in the Western Interconnection (WECC). This slow development is in part due to the high installation cost and in part due to the time delays in getting approval, which have limited their applications for wide area control and stability analysis of power system.

As a member of the PMU family, the Frequency Disturbance Recorder (FDR) was developed at Virginia Tech in 2003. FDR can be used at any $110 \mathrm{~V}$ wall outlet and transmit measured frequency data remotely via the Ethernet. Based on these low cost FDRs, a US-wide Frequency Network (FNET) has been implemented and some power system monitoring applications are being developed by taking full advantage of the FDRs [3], [18], [27], [35]. Thus far there are about 80 FDRs installed in the United States and another 20 installed worldwide. A wide-area Frequency Monitoring Network (FNET) was constructed by utilizing the FDR data collected from the three interconnections in North America. FNET has served utilities, academics and regulators since 2004 with valuable synchrophasor data. Currently, the system is operated by the University of Tennessee and Virginia Tech as a joint effort.

The fact that the FDRs are installed at the $120 \mathrm{~V}$ distribution level reduces their manufacturing and installation costs significantly. For example, at the Tennessee Valley Authority (TVA), the installed cost of one Phasor Measurement Unit (PMU) in 2004 is over $\$ 80,000$. Installed cost of one frequency and angle sensor with GPS and network capability can be made below $\$ 1,000$ each.

\section{B. Event Analysis}

Without loss of generality, we assume the data collected from FNET are 1-D streams. A 3-D data cube can then be constructed with data collected at geographically different locations at a certain moment forming a 2-D image, and data captured at different time moments at the same lo- 
cation forming the 3rd dimension of the data cube. This interpretation facilitates the application of spectral unmixing algorithms in the practice of event unmixing.

Mixed measurements are frequently encountered in realworld applications. Due to the resolution issue associated with discrete sampling and the effect of unknown sources, the measurements can rarely be pure. The wide existence of mixed measurements has brought the decomposition technique a wide array of applications. For example, in the famous cocktail party problem, the listening attention should be focused on a single talker among a mixture of conversations and background noises [2]. In the field of remote sensing, due to the large footprint, a single pixel usually covers more than one type of ground constituent. Thus, the measured spectrum at a single pixel is a mixture of several ground cover spectra, where pixel unmixing has been applied to subpixel object quantification [6], mineral identification [29], area estimation [26], [30], etc. Another emerging application is in biological microscopy where multispectral fluorescence microscopy is analyzed to discriminate different co-localized fluorescent molecules with highly overlapping spectral [11], [15], [31]. Using common microscopy methods, the number of molecules that can be detected simultaneously is limited by both spectral and spatial overlap. These issues can be tackled by applying linear unmixing, which extends the possibilities to distinguish multiple proteins, organelles or functions within a single cell [36].

Similar to the ubiquitous existence of mixed measurements, events rarely occur in a pure and isolated fashion, especially in the power grid. The system disturbance reports from North American Electric Reliability Corporation (NERC) [22] make it obvious that major disturbances typically involve a number of unlikely, "should not have happened", events. Taking the major western blackout in 1996 as an example, at the very beginning of the blackout, two parallel lines were tripped due to a fault and misoperation, and consequently some generation was tripped as a correct special protection system (SPS) response. Then, a third line was disconnected due to bad connectors in a distance relay. After more than 20 seconds of these events, occurred the last straw of the collapse, which was the trip of Mill Creek - Antelope line due to the undesired Zone 3 protective relay. After this tripping, the system collapsed within 3 seconds. Power disturbance, in many ways, exhibits the characteristics of electromechanical wave propagation phenomenon. When multiple disturbances occur in sequence or simultaneously, the electromechanical waves generated will interfere with each other and the measurement taken at an FDR would more than likely be a mixture. Therefore, event unmixing is essential to provide deep insight into system dynamics.

\section{SENSING WITH FNET}

Frequency is a universal parameter across the entire interconnected power system. It can provide information about generation electro-mechanical transients, generation-demand dynamics, and system operations, such as load-shedding, break reclosing, and capacitor bank switching. This characteristic allows frequency monitoring to be as informative at the distribution level as it is at the transmission level.

Frequency computations are usually done from voltage waveform data. Several algorithms [7], [10], [13], [19], [34] have been used, such as zero crossing techniques, least square approximation methods [10], [13], FFT, Kalman Filter methods, and phasor methods [7], [25], [34]. Based on the symmetrical component, and discrete Fourier transform calculations of the positive sequence voltage for a balanced system at a frequency different from the nominal frequency (for instance, $60 \mathrm{~Hz}$ ), a useful result can be obtained to calculate the actual system frequency and phase angle [25].

The most valuable information is that collected during system disturbances. The fast changing nature during system dynamics requires a shorter measurement window to reflect true system status and, as a result, error will increase due to insufficient data, and the computed frequency accuracy will suffer. To capture detailed and fast frequency variations, it has proven necessary in the FNET observation that the accuracy needs to equal or be better than $0.0001 \mathrm{~Hz}$. Higher sampling rate is now necessary. Algorithms that allow significantly higher accuracy and capable of working for a wider frequency range are needed. We adopt the Multiple Re-sampling (MR) technique to increase accuracy a few orders of magnitude from the current design. Fig. 1 illustrates the frequency estimation errors for a $60 \mathrm{~Hz}$ pure sinusoidal waveform with and without the usage of the MR technique.

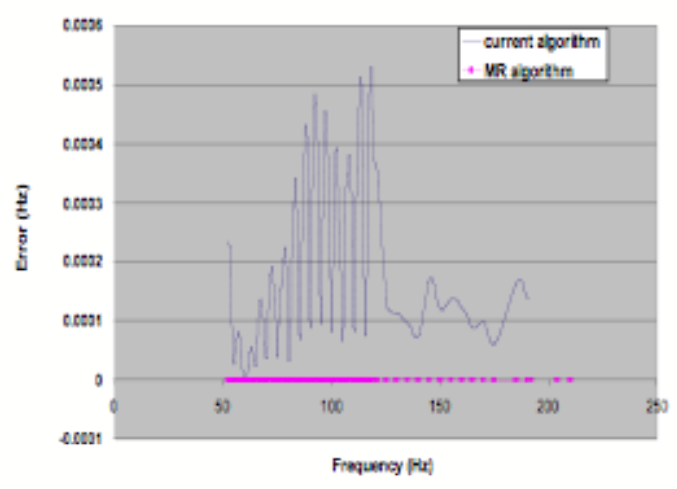

Fig. 1: Frequency estimation errors for a $60 \mathrm{~Hz}$ pure sinusoidal waveform.

Most algorithms discussed so far assume the input voltage waveform is pure sinusoidal. However, in reality, the voltage signal may include noise, harmonic distortions, and sudden changes in magnitude and phase caused by load changes at the distribution level. Many transient conditions occur due to local variations. For example, the voltage waveform of an actual power system may experience amplitude swings from load variations, transient faults, and line switching. Consider the voltage waveform with swing as following:

$$
X(t)=\sqrt{2} X \sin (2 \pi f t)+\sqrt{2} X m \sin \left(2 \pi f_{m} t\right) \sin (2 \pi f t)
$$


where $X$ is the root mean square value of the voltage signal, $f$ is the fundamental frequency, $m$ is modulation index, and $f_{m}$ is the modulation frequency. Typically, in a real system the modulation index will be less than $10 \%$, while the modulation frequency will be around $1 \mathrm{~Hz}$. Without loss of generality, Fig. 2 shows the estimation error - with amplitude swing based on voltage waveform of $5 \%$ modulation and 1 $\mathrm{Hz}$ modulation frequency.

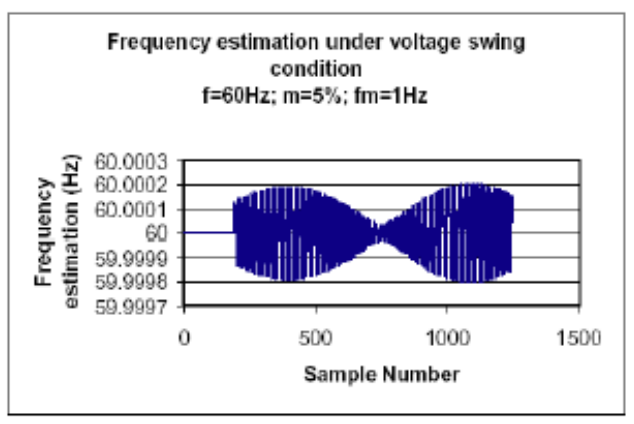

Fig. 2: Estimated frequency from a $60 \mathrm{~Hz}$ signal with amplitude swing.

A sudden phase shift in the voltage waveform will cause similar errors as the amplitude changes. One can first think of the phase shift as being represented by uneven sampling intervals. The solution to this problem lies in the ability to reconstruct the true signal, thus, eliminating phase shift effects as in Fig. 3. However, the basic assumption underlying the reconstruction algorithm is that input is a pure sinusoidal waveform except for the phase shift itself. As noted earlier, the voltage waveform is always contaminated by harmonics and other noise. To maintain the performance of the reconstruction algorithm on a polluted voltage wave remains as a major challenge.

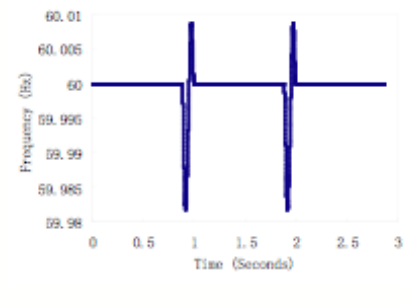

(a) No signal reconstruction

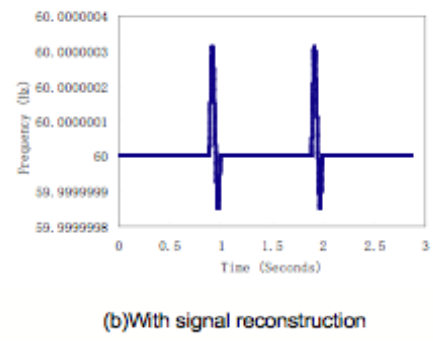

Fig. 3: Frequency estimation errors for a $60 \mathrm{~Hz}$ pure sinusoidal waveform.

\section{EVENT UNMIXING}

High-quality sensing as discussed in Sec. II paves the way to successful data analysis. In this section, we focus on the understanding of events at a finer grain or higher resolution based on the frequency calculated from FNET. The ubiquitous existence of mixed measurements are contributed from multiple events occurring simultaneously or in sequence. Identifying the constituent events, or root events, from the mixture is essential to accurately understand the state of the grid.

In the following, we first formulate the mixing process using the simplest model, the linear mixing model. We then discuss how this can be applied to event unmixing.

\section{A. Problem Formulation}

Linear mixture analysis has been widely used due to its effectiveness and simplicity. Suppose the sensor readout at a single location is given by

$$
\mathbf{x}=\mathbf{A} \mathbf{s}+\mathbf{n}
$$

where the elements of $\mathbf{x} \in \mathbf{R}^{l}$ are the measured mixture or observation. $\mathbf{A}=\left(\mathbf{a}_{1}, \mathbf{a}_{2}, \cdots, \mathbf{a}_{c}\right)$ is an $l \times c$ source matrix (or root event signature matrix) with each column $\mathbf{a}_{j}$ being the signature of one of the root events $j$. The abundance vector $\mathbf{s} \in \mathbf{R}^{c}$ consists of the mixing coefficients satisfying two physical constraints, $s_{j} \geq 0$ (non-negative) and $\sum_{j=1}^{c} s_{j}=1$ (sum-to-one), and $c$ is the number of root events. The last term $\mathbf{n}$ takes into account possible errors and sensor noises. It should be pointed out that together with the constraints, the sensor measurement $\mathbf{x}$ is actually a convex combination of root event signatures; that is, in the $c-1$ hyperspace, all the mixtures are within a simplex, whose vertices correspond to the root event signatures [4], [9].

Given the model in Eq. 1 and assuming the signature matrix is known a priori, the problem of unmixing becomes a constrained linear regression problem. One popular solution is the fully constrained least squares (FCLS) method [14]. The objective is to minimize the least squares error

$$
\begin{array}{ll}
\operatorname{minimize} & f_{0}(\mathbf{s})=(\tilde{\mathbf{x}}-\tilde{\mathbf{A}} \mathbf{s})^{T}(\tilde{\mathbf{x}}-\tilde{\mathbf{A}} \mathbf{s}) \\
\text { subject to } & s_{j} \geq 0, j=1, \ldots, c
\end{array}
$$

where $\tilde{\mathbf{X}}$ and $\tilde{\mathbf{A}}$ are the augmented matrices

$$
\tilde{\mathbf{x}}=\left[\begin{array}{c}
\delta \mathbf{x} \\
1
\end{array}\right], \quad \tilde{\mathbf{A}}=\left[\begin{array}{c}
\delta \mathbf{A} \\
\mathbf{1}^{T}
\end{array}\right]
$$

with $\delta$ being a small weight (typically $1 \times 10^{-5}$ ) and $\mathbf{1}^{T}$ a row vector of all $1 \mathrm{~s}$. This augmentation is used to incorporate the sum-to-one constraint. Note that the constrained minimization problem above is actually the conventional nonnegative least squares problem, which is solved by a standard active set method.

\section{B. The Unsupervised Gradient-Descent Maximum Entropy (GDME) Learning}

Instead of the least squares approach, we develop the maximum entropy principle [21] to formulate the following minimization problem,

$$
\begin{array}{ll}
\operatorname{minimize} & f_{0}(\mathbf{s})=\sum_{j=1}^{c} s_{j} \ln s_{j} \\
\text { subject to } & h_{0}(\mathbf{s})=\mathbf{1}^{T} \mathbf{s}-1=0, \\
h_{i}(\mathbf{s}) & =\sum_{j=1}^{c} a_{i j} s_{j}-x_{i}=0, i=1, \ldots, l,
\end{array}
$$


where the domain of $f_{0}$ is the positive orthant $\mathbf{R}_{++}^{c}=\{\mathbf{s} \in$ $\left.\mathbf{R}^{c} \mid s_{j}>0, j=1, \ldots, c\right\}$. The two equality constraints correspond to the sum-to-one constraint, $h_{0}(\mathbf{s})$, and the sensor measurement model in Eq. $1, h_{i}(\mathbf{s})$. Using the method of Lagrange multipliers, we can easily transform the original constrained minimization problem to an unconstrained problem with the following objective function

$$
\mathcal{L}\left(\mathbf{s}, \boldsymbol{\lambda}, \lambda_{0}\right)=f_{0}(\mathbf{s})+\boldsymbol{\lambda}^{T} \mathbf{h}(\mathbf{s})+\left(\lambda_{0}-1\right) h_{0}(\mathbf{s})
$$

where $\boldsymbol{\lambda}$, and $\lambda_{0}$ are the Lagrange multipliers and $\mathbf{h}(\mathbf{s})$ is a column vector with the $i$ th element being $h_{i}(\mathbf{s})$. This Lagrange function is minimized with respect to $s, \lambda$, and $\lambda_{0}$. However, these three parameters are not independent. Both the abundance vector $\mathbf{s}$ and the multiplier $\lambda_{0}$ can be expressed as a function of $\boldsymbol{\lambda}$. Thus, the minimization problem can be tailored to the finding of the optimal $\lambda$. It can be proved [21] that the analytical solution to the abundance fraction $\mathbf{s}$ obtained by maximizing the Shannon entropy has the classic McCullough-Pitt sigmoid logic in terms of the Lagrange multiplier:

$$
s_{j}=\frac{1}{1+\sum_{\substack{k=1 \\ k \neq j}}^{c} \exp \left(\mathbf{a}_{j}^{T} \boldsymbol{\lambda}-\mathbf{a}_{k}^{T} \boldsymbol{\lambda}\right)}
$$

where $\mathbf{a}_{j}$ is the $j$ th column of $\mathbf{A}$. Note that the two constraints are incorporated in the abundance solution in a more natural way. It is easy to see from Eq. 6 that the abundances sum to 1 and that they are nonnegative. We take the traditional gradient descent method to solve for $\boldsymbol{\lambda}$. The GDME learning starts from an initial $\boldsymbol{\lambda}=\mathbf{0}$, which results in equal abundance distribution based on Eq. 6, i.e., the least biased estimation without learning. It then iteratively solves for $\mathbf{s}$ and $\boldsymbol{\lambda}=\mathbf{0}$ until a stable $\mathbf{s}$ is returned.

We make three important observations comparing the maximum-entropy based minimization and the least-squares minimization. First of all, the underlying principle of using maximum entropy is to incorporate both the non-negative and the sum-to-one constraint in a more natural way. Secondly, GDME and FCLS converge to the same optimal solution, i.e., the least squares estimation. However, GDME starts from a point with uniform abundances and searches within the feasible set, while FCLS takes the unconstrained least squares solution as the initial estimate, and then steers each negative component back to the feasible set. Thirdly, FCLS always gives the least squares solution, which fits the measured data very well. This property is advantageous when the noise level of the measured data is relatively low. If the noise level is high, then the least squares solution actually fits the noisy data, which is not desired. On the other hand, during the convergence of the GDME towards the least squares solution, the fitness is easy to control through appropriate stopping conditions.

\section{Unknown Event Signature and Unsupervised Unmixing}

One of the limitations of the supervised unmixing is that it assumes prior knowledge of the root event signature, making it inefficient in identifying new events. We develop

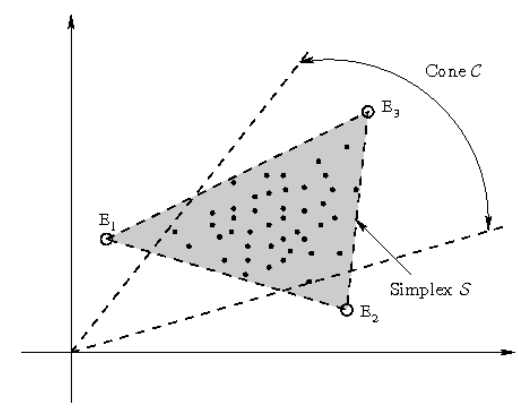

Fig. 4: Geometric illustration of possible cones and simplexes that circumscribe the given data denoted by the dots. The circles $E_{1} \sim E_{3}$ can be considered as possible root events.

another constrained optimization problem [20], based on non-negative matrix factorization (NMF).

As a blind source separation method, NMF has been adopted to solve the problem of mixure decomposition [17], [23], [24]. Given a non-negative matrix $\mathbf{Y} \in \mathbf{R}^{m \times n}$ and a positive integer $r<\min (m, n)$, the task of NMF is to find two matrices $\mathbf{W} \in \mathbf{R}^{m \times r}$ and $\mathbf{H} \in \mathbf{R}^{r \times n}$ with non-negative elements such that

$$
\mathbf{Y} \approx \mathbf{W H}
$$

Suppose any data sample can be thought of as a point in an $l$-dimensional space, whose $l$ coordinates are given by the reflectance values at different spectral bands or frequency readings at different time, the factorization in Eq. 7 reveals that, except for the original Euclidean coordinate system $\left\{\mathbf{e}_{j}\right\}_{j=1}^{l} \in \mathbf{R}^{l}$ (only one element of $\mathbf{e}_{j}$ is 1 , all the others are zeros), there exist other sets of basis vectors $\left\{\mathbf{v}_{j}\right\}_{j=1}^{c}, \mathbf{v}_{j} \succeq \mathbf{0}$ in a subspace $\mathbf{R}^{c}, c<l$, such that all the sample data can be approximated as linear combinations of these bases. As analyzed in [12], for the problem of NMF, all data points lie in a positive simplical cone represented by $\mathcal{C}=\left\{\mathbf{x} \mid \mathbf{x}=\sum_{j} \theta_{j} \mathbf{v}_{j}, \boldsymbol{\theta} \succeq \mathbf{0}\right\}$. However, the extra abundance sum-to-one constraint in the unmixing model confines the data points to reside within a simplex, $\mathcal{S}=$ $\left\{\mathbf{x} \mid \mathbf{x}=\sum_{j} \theta_{j} \mathbf{v}_{j}, \boldsymbol{\theta} \succeq \mathbf{0}, \mathbf{1}^{T} \boldsymbol{\theta}=1\right\}$, where $\boldsymbol{\theta}$ is a column vector with its components being the weight of the basis vectors.

Fig. 4 illustrates one of the possible cones and simplexes for the given data denoted. One immediate and critical question would be what is the criterion for the best simplex solution.

In the convex geometry-based unmixing algorithms, the best simplex is either defined as the one that circumscribes the data cloud and at the same time has the minimum volume [9], or defined as the one that inscribes the data cloud with the maximum volume [33]. In order to find the root event locations from the highly mixed data, we have to extend the searching space outside the given data cloud. In the meanwhile, we should keep the simplex that circumscribes the data as compact as possible. Combining the goal of minimum approximation error with the volume constraint, 
we arrive at the following constrained optimization problem

$$
\begin{array}{ll}
\text { minimize } & f(\mathbf{A}, \mathbf{S})=\frac{1}{2}\|\mathbf{X}-\mathbf{A S}\|_{F}^{2}+\lambda J(\mathbf{A}) \\
\text { subject to } & \mathbf{A} \succeq \mathbf{0}, \mathbf{S} \succeq \mathbf{0}, \mathbf{1}_{c}^{T} \mathbf{S}=\mathbf{1}_{N}^{T}
\end{array}
$$

where $\mathbf{1}_{c}\left(\mathbf{1}_{N}\right)$ is a $c(N)$-dimensional column vector of all 1 's, and $J(\mathbf{A})$ is the penalty function, calculating the simplex volume determined by the estimated endmembers. The regularization parameter $\lambda \in \mathbf{R}$ is used to control the tradeoff between the accurate reconstruction and the volume constraint. The first term serves as the external force to drive the search to move outward, so that the generated simplex contains all data points with relatively small errors. The second term serves as the internal force, which constrains the simplex volume to be small. A solution is found when these two forces balance each other.

One of the expected advantages of the volume constrained NMF would be the resistance to the presence of noise. The noise normally results in a bigger data cloud, which thereby leads to a simplex with larger volume than the clean data, and the simplex vertices deviate from the true endmember locations. By adding the volume constraint, the simplex can be made not to include every data point, particularly, the noisy pixels on the boundary. Therefore, the algorithm would be more robust to the noise effect than the unconstrained NMF approaches.

\section{EXPERIMENTS AND RESULTS}

Generally speaking, there are five root events that would cause disturbances to the power grid, namely, generator trip (event 1), load drop (event 2), line trip type I (event 3), line trip type II (event 4), and oscillations (event 5), as shown in Fig. 5.

To simulate linear mixtures, we create a data cube with each plane of the cube the synchronized readout from different FDRs. Assume FDRs are uniformly and densely deployed, the data cube actualy becomes a multi-spectral image except that the $3 \mathrm{rd}$ dimension is not spectral response but frequency readings over time. We divide the entire image (64-by-64) into small 8-by-8 blocks. The pixels within each block are pure, with the same type of root events randomly assigned. The resulting image is then degraded by a spatial low pass filter to produce mixed pixels. The low pass filter we used is a simple $k \times k$ average filter. Apparently, the value of $k$ controls the degree of mixing. To simulate possible errors and sensor noise, we add zero mean Gaussian random noise to the mixture data.

Both GDME and the NMF-based unmixing algorithms are applied to try to extract the root event signature. GDME assume the existent of "pure" pixel in the data cube although it does not have the knowledge of where exactly they are. However, the NMF-based algorithm does not make any assumption regarding "pure" pixels.

Figs. 6 and 7 show the extracted endmembers from GDME without and with preprocessing of noise depression, respectively. We observe that the unmixing result after preprocessing shows almost exact match with the root event signatures.
The unmixing results from NMF-based algorithm is shown in Fig. 8.

We observe that the signatures extracted from NMF-based algorithm is not as accurate as GDME, which is expected, as GDME makes the more rigid assumption that some pure pixels would be present in the data cube while NMF-based algorithm is completely blind.

\section{Discussions And Future Work}

This paper presented an innovative interpretation of the event formation process, through which high-resolution situational awareness of the power grid based on data collected from FNET would be feasible. We formulated the problem of event unmixing using unsupervised criteria and showed a proof-of-concept simulation. Since wide-area monitoring of the power grid is still at its early stage, most events captured so far only reflect simple scenarios. More realistic and complex scenarios will need to be studied and simulated to further evaluate the effectiveness of the event unmixing algorithms. For example, Fig. 9 illustrates the abundance maps of two root events, generator trip (left) and line trip (right), where the darker the pixel, the larger the abundance of that event in forming the frequency readout from an FDR. The two figures show a typical cascading disturbance to the grid where a generator at the lower-left corner of the map is tripped, causing the line trip nearby. The difference is that the effect of generator trip is propagated throughout the geographical area while the effect of line trip is kept mostly local. Future work will be conducted to realistically simulate the scenario where the two events occur in cascading fashion and effects mixed over time.

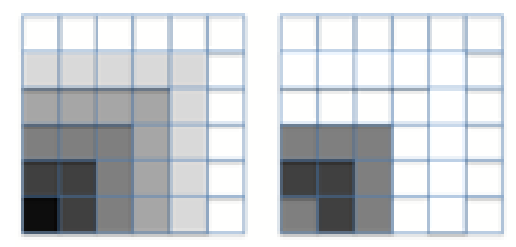

Fig. 9: Conceptual abundance map of two root events. Left: Generator trip. Right: Line trip.

The key to the feasibility of the unmixing process is if a 3D data cube can be formed online and unmixed online. There are actually two ways to form this 3D data cube. One is to take readings from different FDRs as discussed before. The other is to take segments of readings from the same FDR. The latter cannot infer spatial information on how events unravel but reveals temporal information on how a specific location is affected by a sequence of events over time. Another advantage of the latter is that it is a truly localized operation, although the response time might be slower as it needs to wait until enough segments of readings are collected to start computation. This will be another focus of the future work.

\section{REFERENCES}

[1] Synchronized Phasor Measurements and Their Applications. New York: Springer, 2008. 


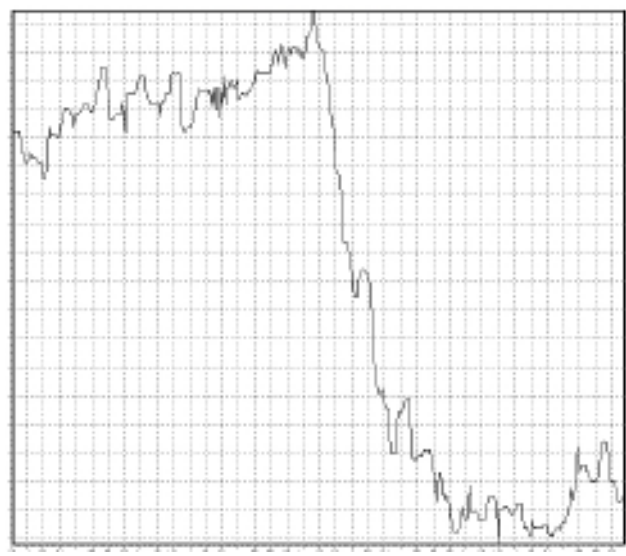

(a) Generator trip.

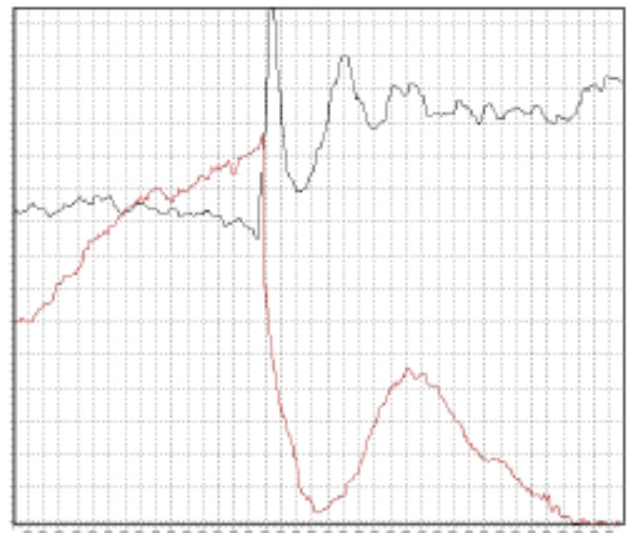

(c) Line trips.

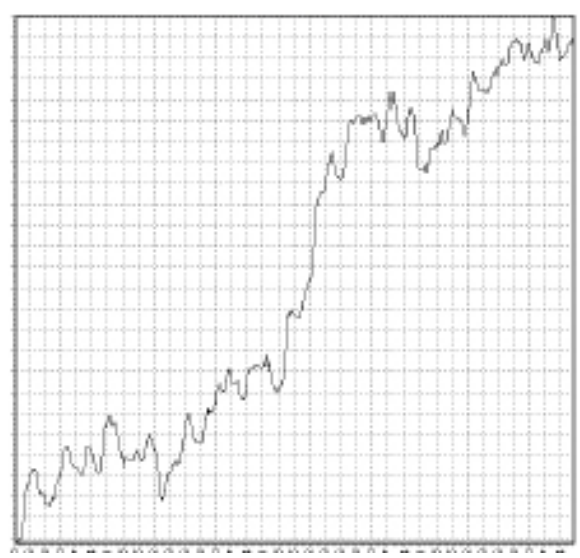

(b) Load drop.

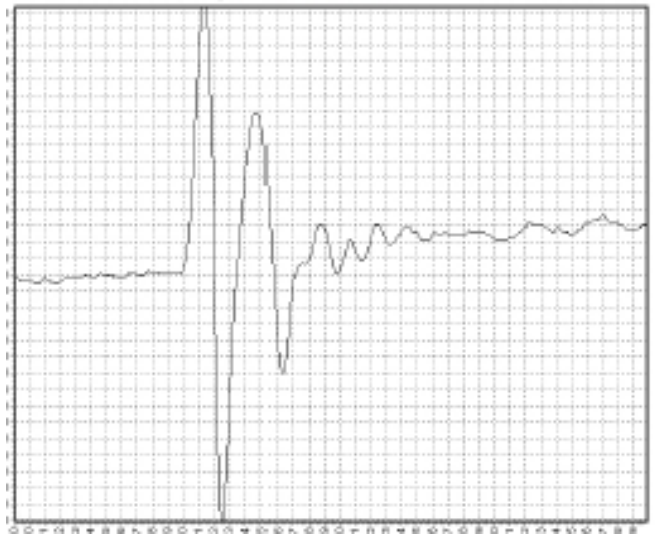

(d) Oscillations.

Fig. 5: Root event signatures.
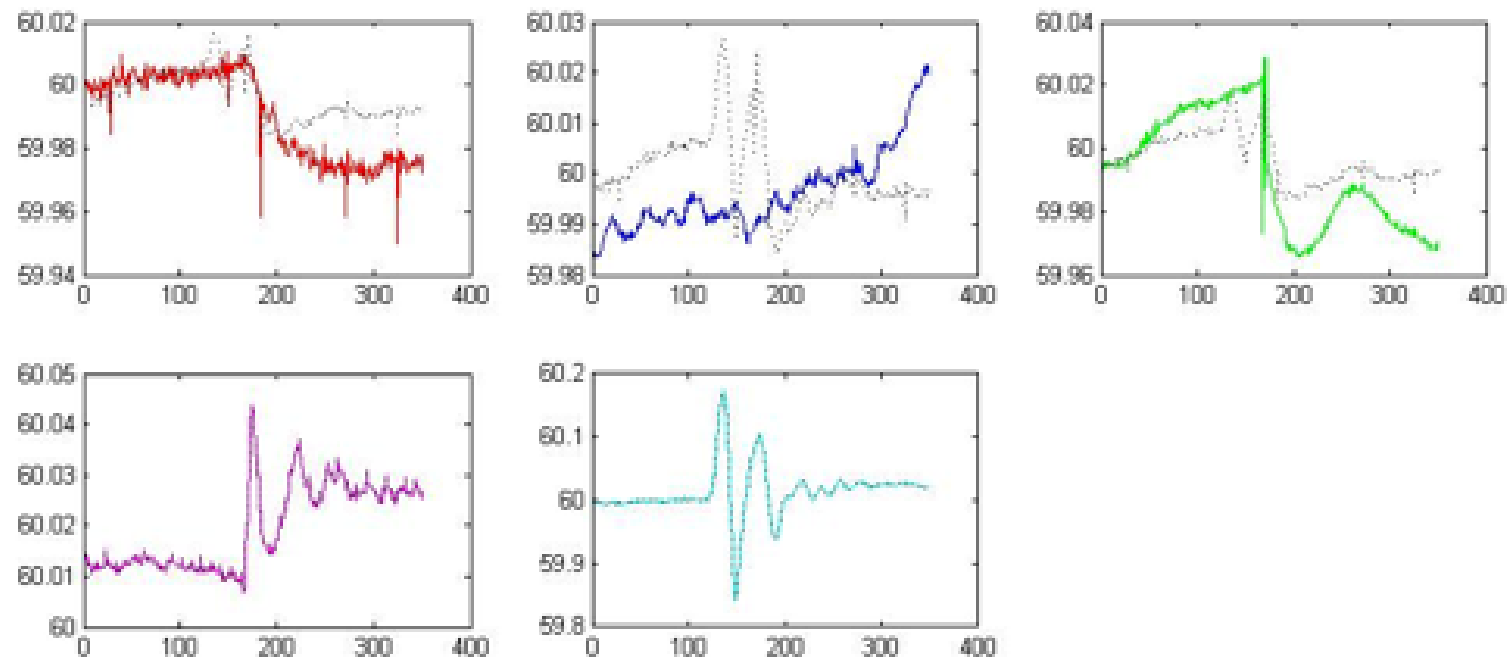

Fig. 6: Root event signatures identified by GDME without preprocessing. 

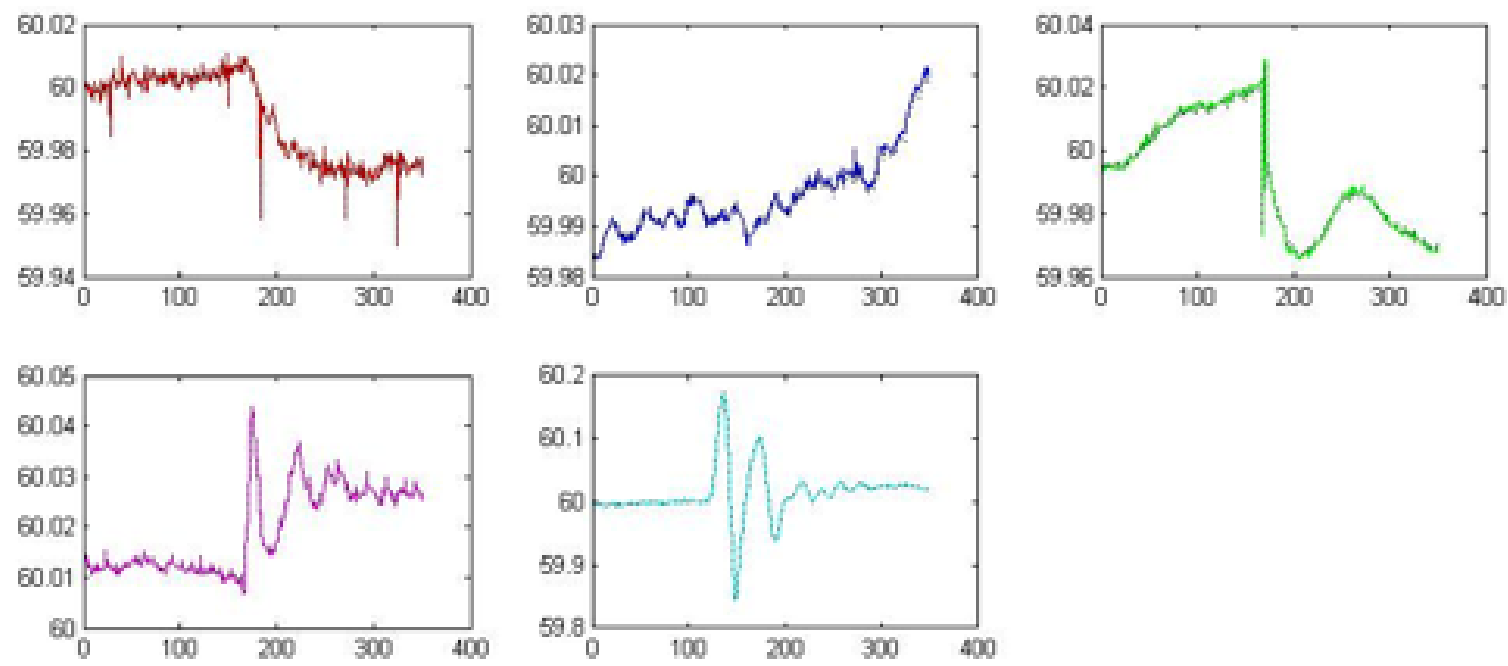

Fig. 7: Root event signatures identified by GDME after preprocessing.
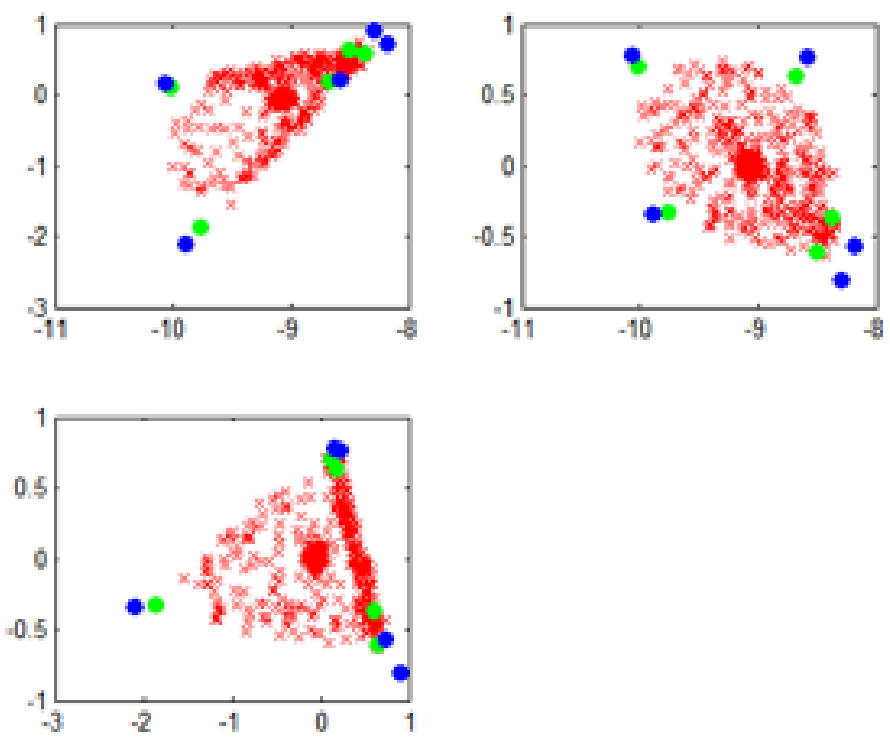

Fig. 8: Comparison between true root event signatures (in green) and estimated signatures (in blue) using NMF-based algorithm with different parameter setup.

[2] B. Arons. A review of the cocktail party effect. Technical report, MIT Media Lab, 1992.

[3] Q. Bin, L. Chen, V. Centeno, Y. Liu, and so on. Internet based frequency monitoring network (fnet). In IEEE Power Engineering Society Winter Meeting, pages 1166-1171, 2001.

[4] J. W. Boardman, F. A. Kruse, and R. O. Green. Mapping target signatures via partial unmixing of aviris data. In R. O. Green, editor, Summaries of Fifth Annual JPL Airborne Earth Science Workshop, volume 1, pages 23-26, 1995.

[5] P. Bonanomi. Phase angle measurements with synchronized clocksprinciple and applications. IEEE Trans. Power Apparatus and Systems, PAS-100(12):5036-5043, Dec. 1981.

[6] C.-I. Chang and D. C. Heinz. Constrained subpixel target detection for remotely sensed imagery. IEEE Trans. Geosci. Remote Sensing, 38(3):1144-1159, May 2000.

[7] J. Chen. Accurate frequency estimation with phasor angles. Master's thesis, Virginia Tech, 1994

[8] J. Chow, L. Vanfretti, A. Armenia, S. Ghiocel, and so on. Preliminary synchronized phasor data analysis of disturbance events in the us eastern interconnection. In IEEE Power Engineering Society Power
Systems Conference and Exposition, pages 1-8, 2009.

[9] M. D. Craig. Minimum-volume transforms for remotely sensed data. IEEE Trans. Geosci. Remote Sensing, 32(3):542-552, May 1994.

[10] P. Dash and A. Pradhan. Frequency estimation of distorted power dystem signal using extended complex kalman filter. IEEE Transactions on Power systems, 14(3):761-765, July 1999.

[11] M. E. Dickinson, G. Bearman, S. Tille, R. Lansford, and S. E. Fraser. Multi-spectral imaging and linear unmixing add a whole new dimension to laser scanning fluorescence microscopy. BioImaging, 31(6):1272-1278, 2001.

[12] D. Donoho and V. Stodden. When does non-negative matrix factorization give a correct decomposition into parts. In S. Thrun and B. Scholkopf, editors, Advances in Neural Information Processing Systems 16, Cambridge, MA, 2004. MIT Press.

[13] A. A. Girgis and W. L. Peterson. Adaptive estimation of power system frequency deviation and its rate of change for calculation sudden power system overload. IEEE Transactions on Power Delivery, 5(2):585-590, April 1990.

[14] D. C. Heinz and C.-I. Chang. Fully constrained least squares linear spectral mixture analysis method for material quantification in hyper- 
spectral imagery. IEEE Trans. Geosci. Remote Sensing, 39(3):529545, Mar. 2001.

[15] Y. Hiraoka, T. Shimi, and T. Haraguchi. Multispectral imaging fluorescence microscopy for living cells. Cell Structure and Function, 27:367-374, 2002.

[16] H. Karimi and so on. Estimation of frequency and its rate of change for applications in power systems. IEEE Trans. on Power Delivery, 19(2):472-480, April 2004.

[17] C.-Y. Liou and K. O. Yang. Unsupervised classification of remote sensing imagery with non-negative matrix factorization. In ICONIP, 2005.

[18] Y. Liu. A us-wide power systems frequency monitoring network. In IEEE Power Engineering Society General Meeting, page 8, 2006.

[19] T. Lobos. Real time determination of power system frequency. IEEE Transactions on Instrumentation and Measurement, 46(4):877-881, Aug. 1997.

[20] L. Miao and H. Qi. Endmember extraction from highly mixed data using minimum volume constrained non-negative matrix factorization. IEEE Trans. on Geoscience and Remote Sensing, 45(3):765-777, March 2007.

[21] L. Miao and H. Qi. A maximum entropy approach to unsupervised mixed pixel decomposition. IEEE Trans. on Image Processing, 16(4):1008-1021, April 2007.

[22] N. A. E. R. C. (NERC). Event analysis: System disturbance reports. http: / / www. nerc. com.

[23] L. C. P. P. Sajda, S. Du. Recovery of constituent spectra using nonnegative matrix factorization. In Proceedings of SPIE, volume 5207 , 2003.

[24] V. P. Paura, J. Piper, and R. J. Plemmons. Nonnegative matrix factorization for spectral data analysis. To appear in Linear Algebra and Applications, 2006.

[25] A. Phadke and J. Thorp. A new measurement technique for tracking voltage phasors, local system frequency, and rate of change of frequency. IEEE Transaction on Power Apparatus and Systems, PAS102(5):1025-1038, May 1983.

[26] N. Quarmby, J. Townshend, J. Settle, K. White, M. Milnes, T. Hindle, and N. Silleos. Linear mixture modelling applied to avhrr data for crop area estimation. Int. J. of Remote Sensing, 13(3):415-425, 1992.

[27] Y. L. R.M. Gardener. Fnet: a quickly deployable and economic system to monitor the electric grid. In IEEE Conference on Technologies for Homeland Security, pages 209-214, 2007.

[28] Y. Shin, A. Parsons, E. Powers, and W. Grady. Time-frequency analysis of power system disturbance signals for power quality. In IEEE Power Engineering Society Summer Meeting, page 402407 , 1999.

[29] M. O. Smith, P. E. Johnson, and J. B. Adams. Quantitative determination of mineral types and abundances from reflectance spectral using principal components analysis. J. Geophys. Res., (90):C797-C804, 1985.

[30] G. Thomas, S. Hobbs, and M. Dufour. Woodland area estimation by spectral mixing: applying a goodness-of-fit solution method. Int. J. Remote Sensing, 17(2):291-301, 1996.

[31] H. Tsurui, H. Nishimura, S. Hattori, S. Hirose, K. Okumura, and T. Shirai. Seven-color fluorescence imaging of tissue samples based on fourier spectroscopy and singular value decomposition. Journal of Histochemistry \& Cytochemistry, 48(5):653-662, 2000.

[32] V. Venkatasubramanian, H. Schattler, and J. Zaborszky. Fast timevarying phasor analysis in the balanced three-phase large electric power system. IEEE Trans. Automatic Control, 40(11):1975-1982, Nov. 1995.

[33] M. E. Winter. Fast autonomous spectral endmember determination in hyperspectral data. In Proc. of 13th international conference on applied geologic remote sensing, volume 2, pages 337-344, Vancouver, BC, Canada, 1999.

[34] X. Zhang. High precision dynamic power system frequency estimation algorithm based on phasor approach. Master's thesis, Virginia Tech, 2004.

[35] Z. Zhong, C. Xu, B. Billian, L. Zhang, and so on. Power system frequency monitoring network (fnet) implementation. IEEE Trans. Power Systems, 20(4):1914-1921, Nov. 2005.

[36] T. Zimmermann, J. Rietdorf, and R. Pepperkok. Spectral imaging and its applications in live cell microscopy. Federation of European Biochemical Societies, 546:87-92, 2003. 